\title{
Familial Hypercholesterolemia: Improving Outcomes with Newer Agents
}

\author{
Saumitra Ray ${ }^{1, *}$, Biswarup Sarkar ${ }^{2}$ \\ ${ }^{1}$ Department of Cardiology, Vivekananda Institute of Medical Sciences, Kolkata, India \\ ${ }^{2}$ Department of Cardiology, RG Kar Medical College and Hospital, Kolkata, India
}

Email address:

drsaumitra@yahoo.co.in (S. Ray)

${ }^{*}$ Corresponding author

To cite this article:

Saumitra Ray, Biswarup Sarkar. Familial Hypercholesterolemia: Improving Outcomes with Newer Agents. American Journal of Internal Medicine. Special Issue: Dyslipidemia: Flash Back and Vision Ahead. Vol. 8, No. 6, 2020, pp. 279-284.

doi: 10.11648/j.ajim.20200806.17

Received: September 23, 2020; Accepted: October 15, 2020; Published: November 16, 2020

\begin{abstract}
Familial hypercholesterolemia (FH) is one of the commonest autosomal dominant genetic disorders which affects lipoprotein metabolism in the body, causing thereby severe hypercholesterolemia, mainly contributed by high level of low density lipoprotein cholesterol (LDLc). This causes polyvascular premature atherosclerosis with significant mortality, especially in the homozygous form $(\mathrm{HoFH})$, where affected persons die in their teens. There are well developed diagnostic criteria for FH but the index of suspicion of the physician needs to be high to detect heterozygous FH. As the blood LDLc values are very high, even the highest doses of statins cannot bring down the levels to optimum. Life style management should also be rigorously implemented. Ezetimibe imparts some extra 10 to $15 \%$ reduction of LDLc on top of maximally tolerated dose of statins. PCSK9I agents are recently approved for FH. They reduce LDLc further by $30-50 \%$. The small interfering RNA, inclisiran, reduces LDLc by almost $60 \%$ and has shown clinical benefit in FH patients. Many other newer agents are in the pipe line of development. In extreme cases, plasmapheresis is life saving, but difficult to adopt as a regular long term treatment. Often the absolute target of LDLc level is not achieved even with all available measures, and a more than $50 \%$ reduction from the baseline value is all that can be attained. Early diagnosis by screening of first degree relatives of the index case helps to attain long term clinical benefit.
\end{abstract}

Keywords: Familial Hypercholesterolemia, Premature Polyvascular Atherosclerotic Disease, Statin, Ezetimibe, PCSK9I, Inclisiran

\section{Introduction}

Familial hypercholesterolemia $(\mathrm{FH})$ is a genetic disorder of lipoprotein metabolism characterized by highly elevated plasma total cholesterol levels with detrimental cardiovascular consequences that commence in childhood. Although atherosclerosis due to FH manifests primarily in adulthood, it has a precocious inception as early as the first decade of life. That early treatment of risk factors can reverse the atherosclerotic changes in the arterial system emphasizes the need for prompt detection and treatment of children with this condition. Fagge identified this disorder more than a century ago as a skin ailment, [1] but its correlation with atherosclerosis was first recognized in 1939 by Norwegian physician Carl Muller. [2] However, FH remains under diagnosed till late due to the lack of awareness among doctors.

Many patients with $\mathrm{FH}$ fail to achieve adequate LDL-C reduction with conventional LDL-C-lowering therapies (LLTs). Between 2012 and 2015, the US Food and Drug Administration (FDA) approved four novel LDL-C-lowering agents for use in patients with homozygous $\mathrm{FH}(\mathrm{HoFH})$, heterozygous $\mathrm{FH}(\mathrm{HeFH})$, or both. Following the precedent set by the approval of statins 30 years ago, these agents were initially approved on the basis of their pronounced LDL-C lowering efficacy without definitive evidence of cardiovascular risk reduction. However, because of their cost, access to these novel agents has been an issue for the FH population. 


\section{Genetics of FH}

\subsection{Genetic Variations of FH}

'Familial hypercholesterolemia' represents the phenotypic manifestation of abnormal lipoprotein metabolism caused by a variety of genetic abnormalities. After the seminal discovery by Brown and Goldstein that mutations in the lowdensity lipoprotein receptor (LDLR) was the cause of monogenetic FH, over 1,500mutations of this gene have been detected [3] and these account for more than $80 \%$ of cases of monogenetic FH. Heterozygous $\mathrm{FH}(\mathrm{HeFH})$ has a prevalence of 1 in 500 in the western world. [4] Homozygous FH $(\mathrm{HoFH})$, although uncommon (prevalence is less than one per million in the general population), is a critical condition which commences in the first few years of life.

In addition to the LDLR defect, two other sets of autosomal dominant mutations play a central role in the pathogenesis of $\mathrm{FH}$; one, a defectiveapo-B100 component of LDL, known as familial defective apoB-100 (clinically indistinguishable from heterozygous LDLR mutations). Secondly, a gain of function mutation affecting proprotein convertase subtilisin/kexin 9 (PCSK9) encoded by chromosome1 has also been shown to trigger $\mathrm{FH}$ by negatively modulating LDL receptor expression. Monogenetic FH is primarily an autosomal dominant disorder with greater than $90 \%$ penetrance.

\subsection{Natural and Modified Natural History of FH}

The natural history of FH depends primarily on the degree of functional LDL receptor activity present, and in turn, on LDL-cholesterol levels, resulting in widely varying prognosis even among homozygous individuals.

The extent of atherosclerosis is primarily determined by the degree of LDL elevation and its duration, calculated by the cholesterol year score. [5] Precocious onset of clinically significant atherosclerotic changes are very common and involve multiple vascular beds including coronary, cerebral, and peripheral systems. Studies in the prestart in era indicated poor outcomes in the majority of patients with $\mathrm{HoFH}$, cardiovascular events being the chief cause of morbidity and mortality. Aortic root disease was reported to be the commonest cardiac manifestation followed by coronary artery disease. While some studies in this interval purported a mean survival of 18 years among patients with $\mathrm{HoFH}$, others observed an average survival of 40 years. Among patients with untreated $\mathrm{HeFH}$, coronary artery disease (CAD) develops in about $50 \%$ of males by the age of 50 years and $30 \%$ of females by 60 years. Although CAD appears 10years later in females compared to males, an accelerated development of CAD is observed after menopause.

England in the pre-statin era showed that mortality associated with CAD was increased a 100-fold in the age group of 20-40 years and four-fold in the 40-59 year age group. [6] Among those surviving to the age of 60 years, however, the risk seems akin to that in the general population.

\subsection{Clinical Features and Diagnosis}

Patients with $\mathrm{HeFH}$ are, by and large, asymptomatic in childhood and adolescence and typically diagnosed by screening methods. Their total and LDL-cholesterol levels are characteristically over the 95th centile of the recommended levels and a strong family history corroborates the diagnosis. Some involved persons may have tendon xanthoma or arcus lipidus.

Homozygous or compound $\mathrm{HeFH}$, on the other hand, presents in the 1st decade of life with a distinctive and severe clinical phenotype. The age at presentation depends on the degree of LDL receptor activity, those with the null phenotype $(<2 \%$ LDL receptor activity) tend to present earlier, resulting even in intrauterine death. These patients have primarily dermatological and ocular manifestationstendon xanthomas and interdigital xanthomas are pathognomic of HoFH. Tendon xanthomas necessitate careful palpation of the Achilles, biceps and triceps tendons for early detection. Although tuberous xanthomas, xanthelasma, and corneal arcus appear in conditions other than FH, their occurrence at a younger age should prompt evaluation for $\mathrm{FH}$.

Severe atherosclerosis involving multiple vascular beds, including coronary, cerebral, and peripheral vascular system, manifest in a myriad ways. Though coronary atherosclerosis is frequently the cause of premature death, calcific aortic valve stenosis and aortic root disease, including supravalvular aortic stenosis due to cholesterol and inflammatory cell infiltration, may result in significant morbidity in these patients, often requiring aortic valve and root replacement.

The diagnosis of FH is typically based on elevation of total-, LDL-, and non-HDL-cholesterol above the $95^{\text {th }}$ centile recommended for the age and sex of the patient together with positive family history or identification of a causative mutation. The MEDPED criteria from the United States, [7] the Dutch Lipid Clinic criteria, [8] and the British Simon Broome Registry criteria [9] are validated diagnostic systems in this regard.

\section{Management}

\subsection{Lipid Targets}

Recommendations differ with respect to target lipid levels in pediatric and adolescent patients. National Lipid Association guidelines recommend a target LDL level of $<130 \mathrm{mg} / \mathrm{dl}$ or $>50 \%$ reduction from baseline values. [9] More rigorous targets are proposed in patients with additional risk factors such as diabetes, obesity, and a family history of CVD. Belgian multisocietal guidelines, on the other hand, recommend age-specific targets, LDL level of $<160 \mathrm{mg} / \mathrm{dl}$ or $>30 \%$ reduction from baseline for children aged $10-14$ years, $<130 \mathrm{mg} / \mathrm{dl}$ for 14 and 18 years and $<100 \mathrm{mg} / \mathrm{dl}$ for older than 18 years. [10] Specific dietary manipulations, 
physical activity, limitation of alcohol intake, and total avoidance of tobacco products form an important part in the management of FH.

Recent guidelines recommend a low calorie diet with a total fat intake of $\leq 3 \%$ of the total dietary intake including $<8 \%$ of saturated fat and $<75 \mathrm{mg} / 1,000 \mathrm{kcal}$ cholesterol for these patients. However, dietary restrictions are noted to have a modest effect in lowering lipid levels, with unproven longterm clinical benefits.

\subsection{Drug Therapy - When to Start}

The former guidelines issued by National Heart, Lung, and Blood Institute (NHLBI) advised treatment with bile acid sequestrants, the lowest age recommended for initiation being 10 years. In a recent statement by the American Heart Association, later endorsed by the American Academy of Pediatrics, statins were proposed as first-line drugs and the age of initiation of therapy was lowered to 8 years.

\subsection{Bile Acid Sequestrants}

They bind to bile acids in the intestine, thereby preventing their systemic absorption; this results in a greater conversion of cholesterol to bile acids and an enhanced production of LDL receptors by the liver. Cholestyramine and colestipol were the most frequently used drugs in this class; however, they fell out of favour due to their modest efficacy (10-20\% LDL reduction) and gastrointestinal intolerance. Of late, a novel drug in this class, colesevelam hydrochloride, has been studied in HeFH patients with good tolerability. [11]

\subsection{Statins}

Statins (3-hydroxy-3methyl-glutaryl-CoA reductase inhibitors) are currently the first line of drugs in the treatment of FH in children and adolescents. They inhibit the ratelimiting step in cholesterol synthesis, thus, increasing the expression of LDL receptors, resulting in the rapid clearance of LDL from the blood.

However, they have a restricted role in patients of $\mathrm{HoFH}$ with null phenotype in view of the need for receptor production for their action. Among the various generic statins available, the Food and Drug Administration (FDA) has approved of pravastatin in children over 8 years of age and lovastatin, atorvastatin, and simvastatin above the age of 10 years. [12] Concurrently, they showed excellent efficacy in lipid lowering with a $26.5 \%$ mean relative reduction in LDLcholesterol levels. The apprehension regarding growth disruption by statinsat puberty was allayed, in part, by the paradoxical finding of increased growth in the children treated with the drug.

Creatine phosphokinase (CK) to assess muscle toxicity and aspartate amino transferase (AST), and alanine amino transferase (ALT) to monitor liver toxicity are mandatory prior to initiation of statins. Follow-up measurements must be done 1-3 months after starting the drug and yearly thereafter. Drug therapy should be interrupted when CK levels reach five times and AST and ALT three times over the upper limit of normal; the same drug at a lower dose or a different statin may be introduced after a drug-free interval of 3 months. Other drugs may be tried if the patient does not tolerate statins despite these measures.

\subsection{Ezetimibe}

Ezetimibe is cholesterol absorption inhibitors and acts on Niemann- Pick C1-like protein at the brush border of the small intestinal epithelium. As their mechanism of action is not based on the expression of LDL receptors, they are especially beneficial in the management of HoFH. Clinical trials have displayed their efficacy in reducing LDL levels when used alone or in combination with statins. However, ENHANCE trial demonstrated that ezetimibe added to high dose simvastatin failed to lessen carotid intima medial thickness in spite of a significant diminution in LDL levels. [13] Lastly, a small but significant rise in the incidence of cancer in SEAS trial [14] is a cause for concern. Therefore, additional data are required on clinically significant outcomes as well as safety endpoints before their widespread adoption in pediatric practice. Although USFDA has approved ezetemibe in children over the age of 10years, current guidelines recommend drug initiation before 18 years of age only in patients intolerant to statins and in patients who fail to realize lipid goals with statin monotherapy.

\subsection{Newer Drugs}

\subsubsection{Agents with Regulatory Approval}

\section{i. Lomitapide}

Lomitapide is an orally administered new lipid-lowering agent with a novel method of action. It inhibits the microsomal triglyceride transfer protein (MTP) which is a heterodimeric enzyme expressed primarily in the endoplasmic reticulum of hepatocytes and enterocytes. It mediates the transfer of triglyceride (TG) molecules to nascent apolipoprotein $\mathrm{B}$ (apoB) during the assembly of very low density lipoproteins and chylomicrons to facilitate hepatic TG secretion and intestinal TG absorption. [15] The US FDA has approved its use as an orphan drug in the treatment of HoFH. In a recently published phase 3 dose escalation trial, lomitapide reduced LDL-cholesterol by $50 \%$ in HoFH patients with poorly controlled LDL levels.

Because MTP inhibition impairs intestinal and hepatic secretion of TG-rich apoB-containing lipoproteins, the most common adverse events and reasons for discontinuing lomitapide were dose-dependent GI disturbances, elevation of hepatic transaminases, and accumulation of hepatic fat. GI events occurred in up to $40-90 \%$ of subjects taking lomitapide despite instructions to restrict dietary fat intake.

\section{ii. Mipomersen}

Mipomirsenis a second-generation antisense oligonucleotide that targets apoB-100 mRNA in the liver, creating a substrate for intrahepatic endonucleases that degrade the apoB100 transcript. It is presently under investigation in the therapy of $\mathrm{FH}$.

This drug significantly lowered LDL and lipoprotein (a) 
levels in adults with heterozygous and homozygous hypercholesterolemia in recent phase 3 trials. [16] Although the mean LDL reduction with $200 \mathrm{mg}$ of subcutaneous mipomirsen administered weekly was significant in patients with $\mathrm{HoFH}(-24.7 \%)$ in treatment group and $-3.3 \%$ in the placebo group $(\mathrm{P}=0.0003)$, the response to therapy was inconsistent and compounded by a significant number of nonresponders.

It gained FDA approval for the treatment of HoFH in 2013. ApoB is the key structural component of LDL. The concept of targeting apoB to reduce serum LDL-C emerged from the discovery that loss-of-function $A P O B$ mutations were causally linked to familial hypobetalipoproteinemia, an autosomal dominant condition characterized by very low plasma levels $(<5$ th percentile for age and sex) of LDL-C and apoB. Mipomersen was also initially developed for LDL$\mathrm{C}$ lowering in a broader population, but it was ultimately approved only for the orphan indication of HoFH.

The most frequent side effects of mipomersen include reactions at the site of injection and flu-like symptoms, and probably steatosis. Moreover, their safety in children is not defined. The frequency of injection-site reactions appears to decline over time, which suggests tolerance might develop to this effect.

iii. Monoclonal Antibodies to Proprotein Convertase Subtilisin/Kexin 9 (PCSK9)

Alirocumab and Evolocumab

Serum PCSK9s are proteins which bind to LDL receptors and promote their degradation, thus, raising LDL levels in the blood. A variety of molecular techniques based on terminating the effect of PCSK9 in order to lower LDLcholesterol levels is under investigation, including the development of monoclonal antibodies that bind toPCSK9, antisense nucleotide-based therapy, and small interfering RNAs. However, as this antibody requires some residual LDL receptor for it to function, it is useful only in patients with $\mathrm{HeFH}$ and non-null phenotype HoFH.

The mature PCSK9 is a $-63-\mathrm{kDa}$ serine protease expressed and secreted primarily by the liver. The clinical importance of PCSK9 emerged through a fascinating sequence of human genetic discoveries in the early 2000s that established a causal relationship between PCSK9 activity and ASCVD risk [17]. It was subsequently shown thatPCSK9 blocks LDLR recycling by binding to the LDLR extracellularly and, after receptor mediated endocytosis, trafficking the LDLR to the lysosome for degradation and depleting the LDLR at the cell surface. Two fully humanized agents from this class gained US regulatory approval in 2015 as adjuncts to maximum tolerated LLT in patients with HeFH or established ASCVD who are not at goal for treatment. Evolocumab is also approved for use in $\mathrm{HoFH}$ subjects.

Results from the evolocumab outcomes trial, FOURIER, confirm that the addition of evolocumab to standard LLT significantly lowers cardiovascular events, albeit to a lesser degree than the $-50 \%$ reduction predicted by preliminary outcomes analyses. FOURIER report showed that evolocumab and statins are associated with similar reductions in major vascular events per $40 \mathrm{mg} / \mathrm{dl}$ reduction in LDL-C (statin versus evolocumab, first year: HR 0.88 versus 0.90 ; second year: HR 0.77 versus 0.83 ).

The most common adverse events associated with these agents, injection-site reactions (2-6\%), were generally mild. Upper respiratory tract symptoms occurred at a similar rate in placebo-treated subjects. In response to findings from early anti-PCSK $9 \mathrm{mAb}$ and statin studies, the incidence of newonset diabetes, muscle-related symptoms, and neurocognitive deficit were specifically examined in longer safety assessments.

\subsubsection{Agents in Clinical Development}

i. Agents in Phase III

a) Inclisiran

Inclisiran (formerly ALN-PCSsc) is a small interfering RNA (siRNA) that leads to sequence-specific degradation of the PCSK9 mRNA transcript with significant effects on plasma LDL-C levels comparable to those seen with the PCSK9 mAbs. In healthy volunteers, a single injected dose of ALN-PCSsc led to dose-dependent mean LDL-C reductions of up to $60 \%$ that were maintained for up to 7 months. [18] In this cohort, a two-dose regimen administered at baseline and day 90 led to dose-dependent mean LDL-C reductions of up to $53 \%$ that also remained significant for up to 8 months. The long duration of these effects is of particular interest as it allows for a significantly lower injection frequency, which could improve adherence. Of note, anti-PCSK9 siRNAs have minimal effect on Lp (a) levels.

b) Bempedoic Acid

Bempedoic acid is an orally administered small molecule inhibitor of hepatic ATP citrate lyase (ACL) and an activator of AMP-activated protein kinase. The inhibition of ACL, a key enzyme in intracellular cholesterol biosynthesis, depletes intracellular cholesterol, which upregulates the LDLR and increases LDL-C clearance [19]. The administration of bempedoic acid as monotherapy and in combination with conventional LLT produced dose-dependent LDL-C reductions of $20-30 \%$ in subjects with pure hypercholesterolemia, statin intolerance, and metabolic syndrome. Bempedoic acid is currently being evaluated in several phase IIILDL-C-lowering studies as well as in a cardiovascular outcomes trial in statin-intolerant subjects.

c) Cholesteryl-Ester Transfer Protein (CETP) Inhibitors

Three CETP inhibitors have failed in cardiovascular outcomes trials [20]. The recent failure of evacetrapib was surprising, given that this agent lowered LDL-C by $37 \%$ compared to placebo. A fourth CETP inhibitor, anacetrapib, was found to significantly but modestly reduce major cardiovascular events by $9 \%$ in REVEAL trial. This outcome was due to reduction in LDL-C (mean -17\%) and non-HDL-C (mean $-18 \%$ ) levels. Despite the positive outcome of this trial, the sponsor has decided not to pursue commercialization of anacetrapib. Another CETP inhibitor, dalcetrapib, is being prospectively studied for its effect on cardiovascular outcomes in a genetically defined subpopulation (dal-GenE) for which evidence suggests there may be a benefit. 


\section{ii. Agents in Phase II}

a) Angiopoietin-like protein 3 (ANGPTL3) inhibitors

ANGPTL3 is a secreted hepatic glycoprotein that impairs the plasma clearance of very low-density lipoprotein (VLDL) and high density lipoprotein (HDL) particles by inhibiting lipases (lipoprotein lipase and endothelial lipase) that hydrolyze TG and phospholipids in circulating lipoproteins. ANGPLT3 was identified as a potential therapeutic target because common and rare loss-of-function ANGPTL3 variants in animals and humans are associated with low plasma levels of TG, HDL-C, and importantly LDL-C; how ANGPLT3 inactivation leads to a reduction in plasma LDL-C remains unclear.

Two pharmacological approaches to ANGPLT3 inhibition are currently in clinical development: a humanized monoclonal antibody to ANGPLT3 (evinacumab) and ANGPTL3-specific antisense oligonucleotides. Evinacumab was reported to significantly reduce both LDL-C and TG levels in healthy volunteers in a dose-dependent fashion [21]. Results from a phase II proof-of-concept study in nine HoFH subjects treated with a multiple-dosing regimen of evinacumab showed a mean LDL-C reduction of $49 \%(157$ $\mathrm{mg} / \mathrm{dl}$ ) after 4 weeks. Based on these results, the FDA granted evinacumab orphan drug status for HoFH. An earlyphase clinical trial of an ANGPLT3-specific antisense oligonucleotide reported dose-dependent reductions in LDL$\mathrm{C}$ (up to $23 \%$ reduction) and TGs (up to $63 \%$ reduction).

b) Gemcabene

Gemcabene is a small-molecule oral agent that inhibits hepatic TG and cholesterol production and apoC-III synthesis [22]. Preclinical evidence suggested that gemcabene's LDL$\mathrm{C}$-lowering effects might be mediated independent of the LDLR, which led to interest in developing this agent for $\mathrm{HoFH}$ and gemcabene was awarded orphan drug status for HoFH. Interim 8-week results from $2 \mathrm{HoFH}$ subjects on maximum LLT enrolled in a phase II multiple-ascendingdose study showed dose-dependent reductions in LDL-C of up to $28 \%$, similar to reductions seen in non-HoFH subjects.

iii. Agents in Phase I

a) LDLR gene therapy

Mutations in LDLR are by far the most common cause of $\mathrm{FH}$, so the concept of "replacing" the defective gene with a functional gene has been of great interest for several decades [23]. In a severe hemophilia B cohort, a gene therapy agent (based on recombinant Factor IX delivered to the liver by adeno-associated virus 8 ) has been associated with clinically significant effects that have been sustained for $>4$ years and shown to be safe. A recombinant LDLR-expressing agent using the same vector, AAV8. TBG. hLDLR, has entered a phase I clinical trial. In preclinical testing, this agent led to pronounced and sustained metabolic and atherosclerotic effects in humanized models of HoFH.

In addition to the aforementioned drugs, other classes of drugs like thyroid mimetics (e.g., eprotiromeandsobetirome) and reconstituted high density lipoprotein (rHDL) are currently under research and shows variable efficacy and safety. All the ongoing trials on modern drug therapy of dyslipidemia focus on adult patients and exclude the pediatric population. Given that a majority of these novel therapies are yet unproven with regard to clinical efficacy and safety endpoints, their role is presently confined to that of a lipid apheresis-sparing therapy in patients with $\mathrm{HoFH}$ who have fallen short of their lipid goals. Additional studies in the pediatric population are required.

b) LDL Apheresis

Patients with homozygous and compound $\mathrm{HeFH}$ frequently need LDL apheresis, which has proved to be a very beneficial treatment option to reducing LDL levels by $55-75 \%$. [24]

Commonly used techniques of LDL apheresis include heparin-induced extracorporeal LDL-cholesterol precipitation (HELP), dextran sulfate cellulose adsorption (DSA), double filtration plasmapheresis (DFPP), polyacrylate full blood adsorption (PFBA also known as DALI), and immune adsorption. However the decline is transitory and is associated with a reboun descalation of lipid levels. This rebound is expeditious in patients without FH, slower in those with $\mathrm{HeFH}$ and delayed in patients in $\mathrm{HoFH}$. Weekly to fortnightly sessions are advocated for patients with HoFH, as such episodic sittings have been shown to reduce the degree of rebound and retard the progression of atherosclerosis. Regular apheresis therapy along with medications in patients of HoFH has improved the average life expectancy to over 50 years of age compared to the formerly bleak prognosis of death in the 2 nd or $3^{\text {rd }}$ decade. Despite its established efficacy, lipid apheresis has not yet been widely embraced in clinical practice due to lack of accessibility for the majority of patients, the prohibitive cost involved, the invasive nature of the procedure, and the lack of motivation among patients.

\section{Surgical Options}

Surgical options including ileal bypass and portocaval shunt have been tried in refractory cases. Owing to the significant comorbidities involved and the need for treatment before the onset of clinical atherosclerosis, these never became popular. Recent case reports of successful pediatric liver transplant done for the treatment of HoFH suggest excellent efficacy and good safety profile of this option. [25] However, in view of the scarcity of donor liver available and complexities of the transplant and post-transplant management, such a decision should be taken only after carefully assessing the risk benefit ratio.

\section{Conclusion}

FH is a grave ailment with its genesis in early childhood resulting in damaging consequences in later life. Although the need for a screening strategy to detect this disease early is widely accepted, there is no consensus regarding whom and when to screen. Early initiation of lipid-lowering therapy and lifestyle measures might improve the clinical outcome. While such treatment initiatives have notably improved the prognosis of $\mathrm{HeFH}$, the outcomes of $\mathrm{HoFH}$ remain 
disappointing. Although most cases may be treated with a combination of statins and cholesterol absorption inhibitors, some will have need of more invasive therapies such as LDL apheresis. The past 2 decades have noted the evolution of novel therapies to lower LDL-cholesterol levels and defer premature atherosclerosis, especially in conjunction with lifestyle modifications. Despite these triumphs, a large majority of children do not attain targeted lipid goals due to shortfalls in diagnosis, monitoring, and treatment.

The past decade has witnessed the development of an unprecedented number of highly efficacious LDL-C-lowering agents that have the potential to revolutionize the management of FH. However, whether these agents will have as profound an impact as is hoped will largely be determined by the proportion of FH patients that will be able to access them.

\section{References}

[1] Fagge CH. Xanthomatous diseases of the skin. Trans Pathol Soc Lond $1873 ; 242-50$

[2] Muller C. Angina pectoris in hereditary xanthomatosis. Arch Intern Med 1939; 64: 675-700.

[3] Goldstein JL, Brown MS. Binding and degradation of low density lipoproteins by cultured human fibroblasts. Comparison of cells from a normal subject and from a patient with homozygous familial hypercholesterolemia. J Biol Chem 1974; 249: 5153-62.

[4] Goldstein JL, Hobbs HH, Brown MS. Familial hypercholesterolemia. The metabolic and molecular bases of inherited disease. 8th ed. New York: McGraw-Hill, Medical Publishing Division; 2000. pp. 2863-913.

[5] Raal FJ, Santos RD. Homozygous familial hypercholesterolemia: Current perspectives on diagnosis and treatment. Atherosclerosis 2012; 223: 262-8.

[6] Risk of fatal coronary heart disease in familial hypercholesterolaemia. Scientific Steering Committee on behalf of the Simon Broome Register Group. BMJ1991; 303: 893-6.

[7] Williams RR, Hunt SC, Schumacher MC, et al. Diagnosing heterozygous familial hypercholesterolemia using new practical criteria validated by molecular genetics. Am J Cardiol1993; 72: 171-6.

[8] Civeira F; International Panel on Management of Familial Hypercholesterolemia. Guidelines for the diagnosis and management of heterozygous familial hypercholesterolemia. Atherosclerosis 2004; 173: 55-68.

[9] Daniels SR, Gidding SS, de Ferranti SD; National Lipid Association Expert Panel on Familial Hypercholesterolemia. Pediatric aspects of familial hypercholesterolemias: Recommendations from the National Lipid Association Expert Panel on Familial Hypercholesterolemia. J Clin Lipidol 2011; 5 (3Suppl): S30-7.

[10] Descamps OS, Tenoutasse S, Stephenne X, et al. Management of familial hypercholesterolemia in children and young adults: Consensus paper developed by a panel of lipidologists, cardiologists, paediatricians, nutritionists, gastroenterologists, general practitioners and a patient organization. Atherosclerosis 2011; 218: 272-80.

[11] Stein EA, Marais AD, Szamosi T, et al. Colesevelam hydrochloride: Efficacy and safety in pediatric subjects with heterozygous familial hypercholesterolemia. J Pediatr 2010; 156: 231-6. e1-3.

[12] Lughetti L, Bruzzi P, Predieri B. Evaluation and management of hyperlipidemia in children and adolescents. Curr Opin Pediatr 2010; 22: 485-93.

[13] Kastelein JJP, Akdim F, Stroes ES, et al. Simvastatin with or without ezetimibe in familial hypercholesterolemia. N Engl J Med2008; 358: 1431-43.

[14] Rossebø AB, Pedersen TR, Boman K, et al.; SEAS Investigators. Intensive lipid lowering with simvastatin and ezetimibe in aortic stenosis. N Engl J Med 2008; 359: 134356.

[15] Cuchel M, Bloedon LT, Szapary PO, et al. Inhibition of microsomal triglyceride transfer protein in familial hypercholesterolemia. N EnglJ Med 2007; 356: 148-56.

[16] Stein EA, Dufour R, Gagne C, et al. Apolipoprotein B synthesis inhibition with mipomersen in heterozygous familial hypercholesterolemia: Results of a randomized, double blind, placebo-controlled trial to assess efficacy and safety as add-on therapy in patients with coronary artery disease. Circulation 2012; 126: 2283-92.

[17] Abifadel M, Varret M, Rab'es J-P, et al. 2003. Mutations in PCSK9 cause autosomal dominant hypercholesterolemia. Nat. Genet. 34 (2): 154-56.

[18] Ray KK, Landmesser U, Leiter LA, et al. 2017. Inclisiran in patients at high cardiovascular risk with elevated LDL cholesterol. N. Engl. J. Med. 376 (15): 1430-40.

[19] Pinkosky SL, Newton RS, Day EA, et al. 2016. Liver-specific ATP-citrate lyase inhibition by bempedoic acid decreases LDL-C and attenuates atherosclerosis. Nat. Commun. 7: 13457.

[20] Tall AR, Rader DJ. 2017. The trials and tribulations of CETP inhibitors. Circ. Res. In press.

[21] Dewey FE, Gusarova V, Dunbar RL, et al. 2017. Genetic and pharmacologic inactivation of ANGPTL3and cardiovascular disease. N. Engl. J. Med. 377 (3): 211-21.

[22] Bisgaier CL, Essenburg AD, Barnett BC, et al. 1998. A novel compound that elevates high density lipoprotein and activates the peroxisome proliferator activated receptor. J. Lipid Res. 39 (1): $17-30$.

[23] Ajufo E, Cuchel M. 2016. Recent developments in gene therapy for homozygous familial hypercholesterolemia. Curr. Atheroscler. Rep. 18 (5): 22

[24] Thompson GR; HEART-UK LDL Apheresis Working Group. Recommendations for the use of LDL apheresis. Atherosclerosis 2008; 198: 247-55.

[25] Küçükkartallar T, Yankol Y, Kanmaz T, et al. Liver transplantation as a treatment option for three siblings with homozygous familial hypercholesterolemia. Pediatr Transplant2011; 15: 281-4. 